Research Article

\title{
Path Planning and Trajectory Tracking Strategy of Autonomous Vehicles
}

\author{
Peng Han ${ }^{1}{ }^{1}$ and Bingyu Zhang $\mathbb{D D}^{2}$ \\ ${ }^{1}$ School of Air Traffic Management, Civil Aviation University of China, Tianjin 300300, China \\ ${ }^{2}$ Railway Motive Power Department, Tianjin Railway Technical and Vocational College, Tianjin, China \\ Correspondence should be addressed to Peng Han; tpl_hp@163.com
}

Received 11 September 2020; Revised 8 January 2021; Accepted 21 January 2021; Published 31 January 2021

Academic Editor: Haipeng Peng

Copyright (c) 2021 Peng Han and Bingyu Zhang. This is an open access article distributed under the Creative Commons Attribution License, which permits unrestricted use, distribution, and reproduction in any medium, provided the original work is properly cited.

\begin{abstract}
With the development of global urbanization and the construction of regional urbanization, residents around urban cities are increasingly making demands on urban public transportation system. A new kind of modern public transportation vehicle named Multi-Articulated Guided Vehicle based on Virtual Track (MAAV-VT) with the advantages of beautiful, smart energy conservation and environmental protection is proposed in this paper, which aims at optimizing the public transportation system between and within urban areas. Therefore, concentrating on the general design and control strategy, the main contents of this paper are as follows. At first, the design concepts and key technologies of MAAV-VT are introduced. It is the fusion of urban rail transit operation mode and advanced automotive technologies, which have the characteristics of $100 \%$ low-floor, medium to high velocity, medium to big capacity, and low construction cost. Then, as the core subsystem, to guarantee the properties of selfguiding and trajectory tracking of the new vehicle, this paper is focused on the control system based on the dynamics and kinematics model of the whole multi-articulated vehicle. The multi-trace-points cooperative trajectory tracking control strategy on the basis of the circulation of feasible path generation method is proposed and the lateral controller is designed for trajectory tracking. The process of feasible path generation is conducted once the tracking error exceeded. A simulation platform is built considering the mechanical properties of each vehicle element and the characteristic of articulated mechanism. Finally, the function of control system is validated. The tracking error of each vehicle elements would be reduced to make sure the whole multi-articulated vehicle moves along the preset virtual track.
\end{abstract}

\section{Introduction}

As the rapid growth of private transportation, the construction level of urban road system is hardly to satisfy the demand of transportation which caused the phenomenon of traffic environment deterioration and the imbalance between supply and demand. This is typical in big cities of emerging developing countries. Developing the urban public transportation system energetically is an effective countermeasure to mitigate the transportation press. The guided vehicle system could play an important role in public transportation system. The guided vehicle is guided by external medium and operated without the driver's control [1]. The external medium which plays the role of guidance could be both contactless form such as optics and magnetics and contact form including guide wheel and rail [2]. The "CIVIS" [3] and "Phileas" [4] are two typical guided vehicles which had been used for engineering applications. As well, the AutoTram Extra Grand is also a long guided vehicle developed by the Fraunhofer IVI, combining the advantages of rail and road-bound transport systems. A few similar vehicles and the lines in operation are listed in Table 1.

The advanced automation control technologies in the field of intelligent vehicles, such as motor control technology, navigation technology, identification technology of operation environment, and trajectory tracking technology are developed rapidly in recent years. 
TABLE 1: A few guided vehicles and the lines in operation.

\begin{tabular}{lcc}
\hline Vehicle type & Guidance mode & Lines in operation \\
\hline O-bahn (Mercedes, Volvo) [5] & Physical guidance (guide wheel in side) & Adelaide, Leeds, Essen \\
CIVIS (Iris bus) & Optical guidance & Las vegas, Rouen, Clermont-Ferrand \\
Phileas (APTS) & Magnetic guidance & Eindhoven \\
Stream (Ansaldobreda) & Magnetic guidance & Trieste \\
Aeg (Cegelec) & Induction cable guidance \\
Toer [6] & Optical guidance & Channel tunnel, Shuttle \\
\hline
\end{tabular}

First of all, the guided system and environment perception technology provide the operation lines information and environment information around the bus to the control system. The guided system is mainly constituted by one or more of the navigation methods including GPS navigation, inertial navigation, laser navigation, magnetic navigation, and visual navigation. The researches on environment perception technology are concentrated on the access of the obstacle information and traffic signal information measured by the camera, radar, and other related sensors. Reid [7] proposed an automatic guidance method for tractor by differential dynamic positioning technology with GPS in real time. Will [8], Makela [9] and Bakambu [10] designed the guided systems for agricultural machinery and submarines separately. Chan [11] focused on two types of magnetic systems to identify the characteristics of these two sensing systems and to offer a comparison of their distinct features. Hopstock [12] developed a permanent magnetic pavement marking tape. Sections of varying magnetization wavelength were installed in a 230-m linear array. Magnetic field profiles were determined at lateral displacements from the tape out to $0.9 \mathrm{~m}$. Søgaard [13] developed a laser optic position determination system (PDS) by mounting it on an agricultural tractor and a sowing machine. Se [14] described a kind of vision-based mobile robot localization and mapping algorithm using scale-invariant image features as landmarks in unmodified dynamic environments. Giovanni [15] reviewed three approaches to vision-based self-localization used in the RoboCup middle-size league competition and described the results they achieved in the robot soccer environment for which they had been designed.

The control system of guided vehicle should export accuracy control commands to make the vehicle move along the expected trajectory. The control system integrates these subsystems including information perception, task planning, behavior decision, and execution organization. Reasonable decision support architecture could increase the decision-making ability of the whole system. Several kinds of decision structures are used in intelligent vehicles. Rosenblatt [16] developed a distributed architecture for mobile navigation system. Brooks [17] described subsumption architecture for controlling mobile robots. Layers of control system were built to let the robot operate at increasing levels of competence which are made up of asynchronous modules that communicate over low-bandwidth channels. Higherlevel layers subsumed the roles of lower levels by suppressing their outputs. Other scholars also developed the architecture like hierarchical intelligent control structure [18], multilevel structure system $[19,20]$, and so on $[21,22]$.
The researches on trajectory tracking are very rich at the field of intelligent vehicles. The intelligent vehicles are typically nonholonomic constraint system which has the characteristics of highly nonlinear and complexity. Thus, it is fairly difficult to propose the control strategy based on precise modeling. The commonly used tracking control strategy decouples the lateral and longitudinal motion. Thus, the path following and velocity control of the system could be individually controlled. The path following could be divided into preview control and compensation control. The preview control methods [23-25] calculate the target quantities which control the motion state of the intelligent vehicles first based on the dynamics model and the kinematics model. The real time condition monitoring system should give a feedback of the actual lateral acceleration, yaw velocity, sideslip angle, and so on. The controller makes the difference between the calculated target and actual quantities decrease to approach the target path. Xia [9] proposed a novel approach combining the sliding mode control and extended state observer (ESO) for attitude control of a missile model. Saber [26] developed trajectory tracking and configuration stabilization for the vertical takeoff and landing (VTOL) aircraft which addressed global configuration stabilization for the VTOL aircraft with a strong input coupling using a smooth static state feedback. The compensation control methods monitor the target trajectory and actual location of the vehicle. Then, the actuators which control the motion of the vehicle are controlled directly to make the vehicle move along the trajectory. The steering model, kinematics model, and dynamics model are often used in the control system. The control method could also be classified based on the control strategy, such as PID method, optimum control, sliding-mode control, model predictive control, fuzzy control, and neural network control. All these methods are applied in intelligent vehicle control system and obtained good results.

In recent years, there are some safer and more robust algorithms in the field of path following control, especially for autonomous vehicle. For example, Zhang [27] investigated the path following control problem for four-wheelindependent-drive electric vehicles with consideration of modeling errors and complex driving scenarios which employed a suppress twisting second-order sliding mode (SOSM) control strategy to suppress the heavy chattering issue existing in the traditional sliding mode control (SMC). In addition, this team provided a new solution for path following control of autonomous ground vehicles which formulated a standard model and represented in a TakagiSugeno fuzzy form to deal with the time-varying nature of 
the vehicle speed [28]. To enhance the vehicle safety, this team further investigated the problem of steering actuator fault diagnosis for automated vehicles based on the approach of model-based support vector machine (SVM) classification [29].

The development of these advanced technologies provides proper environment for the development of guided vehicle. On this background, a new vehicle concept based on virtual track which combines the advantages of intelligent guided vehicle and rail transportation system is proposed. In this work, the job designing of multi-articulated guided vehicle is proposed to solve the problem of urban public transportation congestion. The design concepts and general technologies involved in the design process of the new vehicle are proposed. To solve the problem of uncoordinated movement of front and rear carriages during the operation of the multi-articulated vehicle, a collaborative tracking algorithm based on dynamic and kinematic characteristics are proposed. The contributions and highlights of this work are summarized as follows:

(1) Three layers framework of control system including identification and monitoring, feasible trajectory planning, and execution are introduced.

(2) The dynamic and kinematic model between the joint constraints and each carriage is built which ultimately formats the whole characteristic of multiarticulated vehicle.

(3) A feasible path planning method and trajectory tracking strategy of the multi-articulated vehicle is proposed and verified by the constructed simulation platform.

\section{Design Concepts and General Technologies of the MAAV-VT}

The multi-articulated guided vehicle is a new public transportation vehicle which positions as an important component of public transport system. The construction mode is able to take full advantages of the city space and develop a comprehensive transportation system with the connection of other vehicles between and within urban areas. The MAAV-VT could undertake different responsibilities including urban agglomerations transportation and inner-city transportation. It is an extension and supplement of the urban public transport system. The MAAV-VT is the fusion of operation mode of rail transit and automobile emerging technologies. It has the advantages of high velocity and big capacity like railway vehicles. Furthermore, it could operate without steel rail which helps decrease the construction cost and keep the road neat and beautiful. The design concepts of the MAAV-VT are described as the following six parts which are shown in Figure 1.

(1) Multi-articulated connection for big capacity: the new vehicle should have a big carrying capacity of about 15 thousand persons per hour with the operation speed of $40-50 \mathrm{~km} / \mathrm{h}$. In this situation, the guided vehicle should have at least three units for carriage. Thus, the multi-articulated connection method is used here. This characteristic helps the vehicle possess the ability like traditional railway vehicles.

(2) Rubber tyre support to operate without rail: the new vehicle should adopt rubber tyre support mode. This is the guarantee to operate without steel rail. There is no need to destroy the existing road surface in the construction progress. The integrity of the road is reserved which saves a lot cost and keeps the road beautiful.

(3) Virtual track guidance for self-guiding: the lack of steel rail also brings loss of physical constraints of the vehicle. Thus, the guidance function which is provided by steel rail should be reformed. Virtual track guidance here is used for self-guiding. Virtual track is defined as a series of continuous or discrete signal band. It could be set as electronic map, magnetic nail, or vision based band to guide the vehicle.

(4) Specially designed structure and control strategy for trajectory tracking: the structure of the vehicle and the tracking controller should also be specially designed to realize self-guiding. The vehicle elements are connected by articulated mechanism. The trajectory following ability of the whole vehicle should be guaranteed by the specially designed structure and a suitable control strategy.

(5) Hub motors for independent driving: independent driving is an effective method to design $100 \%$ lowfloor vehicle. Hob motors is very suitable here for increasing the traction efficiency and simplifying the structure of the machinery drive system. Even more important, isolated control of each wheel is convenient for the trajectory following of each vehicle element.

(6) Mixed road rights with existing road vehicles to improve efficiency: the vehicle should operate with other road vehicles in unban cities. The synergetic service of all these vehicles leads to great efficiency of urban transport system.

According to the design concepts, a few technologies include the identification and guidance of virtual track, selfguiding, and trajectory tracking and the control strategy of hub motors are combined in order to realize the designation. As the core of the Multi-Articulated Guided Vehicle based on Virtual Track, the control system is the key to guarantee the properties of self-guiding and trajectory tracking. The role of control system and the relationship between it and other subsystems of the MAAV-VT are focused on this paper.

The framework of control system based on multilevel hierarchical theory is shown in Figure 2. There are three layers in the system including identification and monitoring, feasible trajectory planning, and execution to assure the vehicle move along the given virtual track. The input of the control system is the identified results of virtual tracks. 


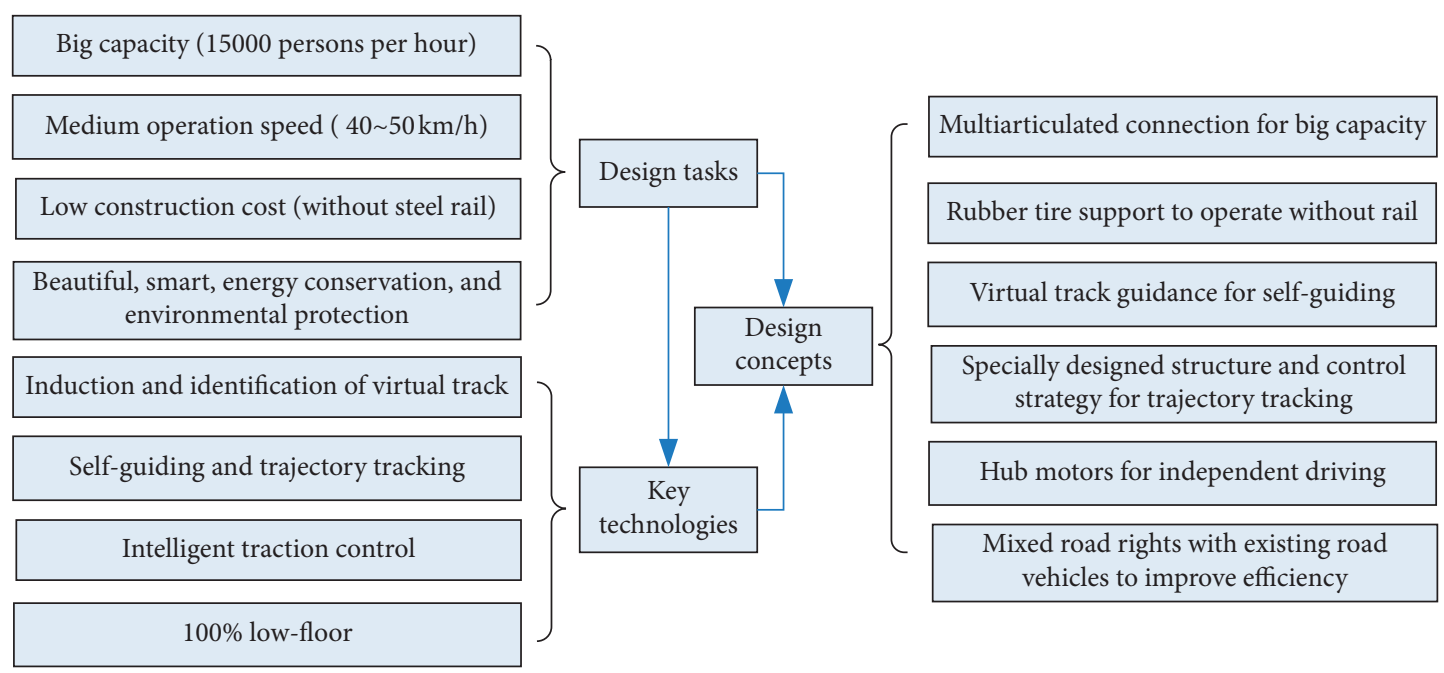

Figure 1: The design tasks and concepts.

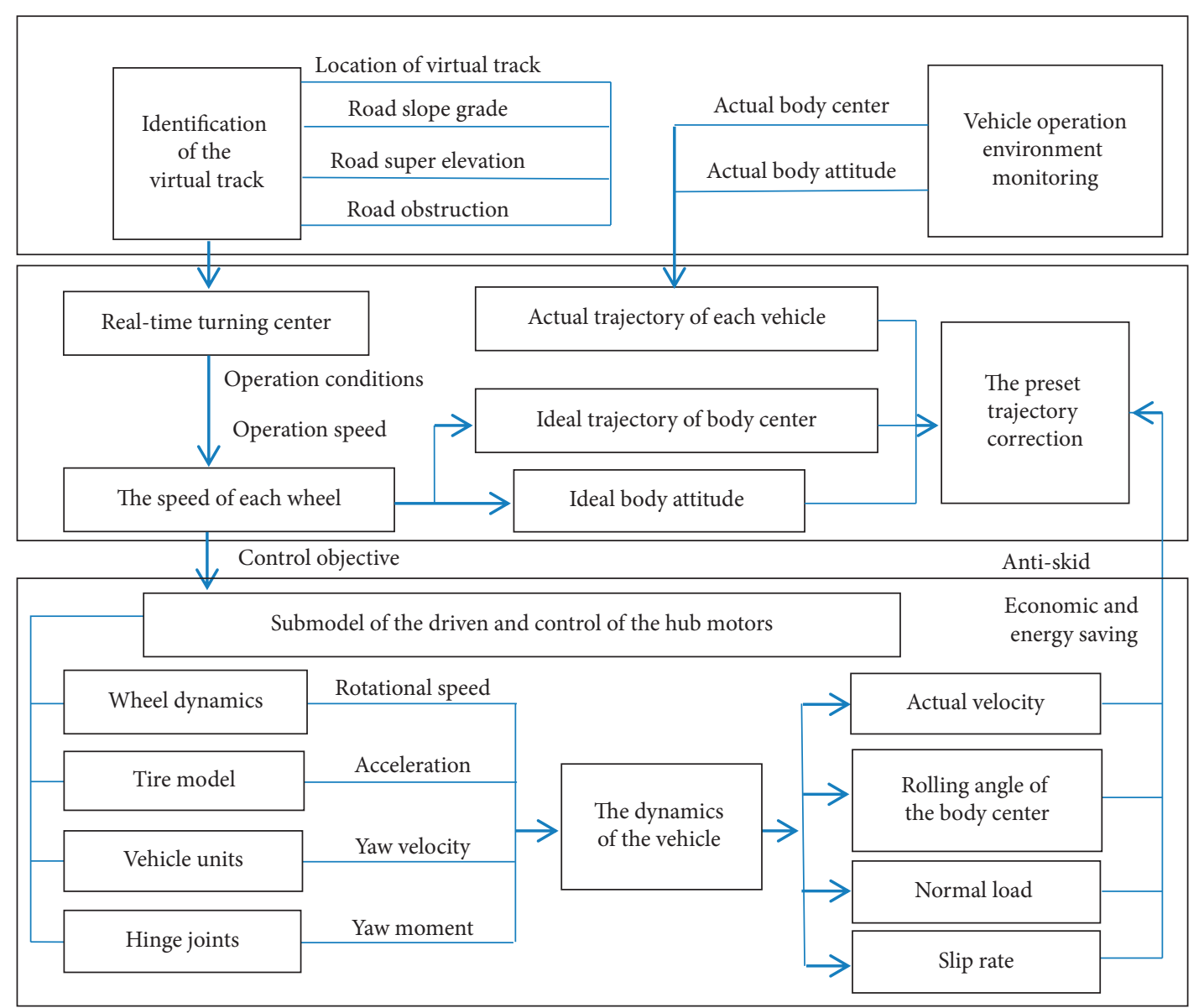

FIGURE 2: The three layers of the control system.

The geometry information of the virtual track is perceived and identified first, such as the location, road slope grade, super elevation, and obstruction. The vehicle's realtime operation status including the attitude and location should also be monitored. Then, feasible trajectory of the whole multi-articulated vehicle is planned according to the boundary conditions of the kinematics and dynamics model. Finally, the execution layer is the key to make sure trajectory tracking and following through the accurately control of each motor. The objective velocity and torque of each wheel for tracking the planned feasible trajectory are calculated separately. The control of hub motors is based on the vehicle 
system dynamics, the friction model between wheel and ground, and the control theory. The three layers formed a closed loop from the identification and monitoring to the trajectory tracking control strategy.

\section{Kinematics Model of MAAV-VT System}

3.1. Description of Virtual Track. The multi-articulated guided vehicle is operated by the guidance of preset virtual track on the road. The virtual track in front and the actual position and attitude of the guided vehicle should be identified based on the optical identification system. A series of coding graphs are used to describe the virtual track. The QR codes have the advantages of uniqueness and veracity. Furthermore, the codes could store the information of virtual track including the location in front, road slope grade, and super elevation. The actual position and attitude of each vehicle element relative to the virtual track could be measured and calculated by the visual system.

3.2. Kinematics Model of Joint Constraints. As is shown in Figure 3, the kinematics constraints are acted between two vehicle elements. The moving coordinate system are denoted as $O_{i} X_{i} Y_{i} Z_{i}$ and $O_{j} X_{j} Y_{j} Z_{j}$. Taking the revolute joint as an example, the revolute joint has limited the three degrees of freedom of parallel motion and the rotational motions of horizontal and longitudinal. Only the vertical rotational motion is retained. The axles of revolution of the two vehicle elements are recorded as $\overrightarrow{\omega_{i}}$ and $\overrightarrow{\omega_{j}}$. Two orthogonal vectors are selected in the second element and recorded as $\overrightarrow{\omega_{j 1}}$ and $\overrightarrow{\omega_{j 2}}$ separately. The equations of kinematics constraints in the hinge point are expressed as follows:

$$
\mathbf{C}\left(\mathbf{q}_{i}, \mathbf{q}_{j}\right)=\left\{\begin{array}{l}
\mathbf{r}_{i J}-\mathbf{r}_{j J} \\
\boldsymbol{\omega}_{i} \cdot \boldsymbol{\omega}_{j 1} \\
\boldsymbol{\omega}_{i} \cdot \boldsymbol{\omega}_{j 2}
\end{array}\right\}=0,
$$

where $\mathbf{r}_{i J}$ and $\mathbf{r}_{j J}$ represent the position vector in the hinge point of the first and second vehicle elements. Substituting the transformation matrix of the ground fixed coordinate and car body following coordinate which named as $\mathbf{A}_{i}$ and $\mathbf{A}_{j}$ into Equation (1), we can get:

$$
\left\{\begin{array}{c}
\mathbf{R}_{i}+\mathbf{A}_{i} \overrightarrow{\mathbf{r}_{i J}}-\left(\mathbf{R}_{j}+\mathbf{A}_{j} \overrightarrow{\mathbf{r}_{j J}}\right) \\
\mathbf{A}_{i} \overrightarrow{\mathbf{\omega}_{i}} \cdot \mathbf{A}_{j} \overrightarrow{\mathbf{\omega}_{j 1}} \\
\mathbf{A}_{i} \overrightarrow{\mathbf{\omega}_{i}} \cdot \mathbf{\mathbf { A } _ { j }} \overrightarrow{\mathbf{\omega}_{j 2}}
\end{array}\right\}=0
$$

where $\mathbf{R}_{i}$ and $\mathbf{R}_{j}$ represent the position vectors of the original point of the two car bodies following coordinates. The Jacob matrix of the constraint equation is expressed as follows.

$$
\dot{C}_{q}\left(\mathbf{q}_{i}, \mathbf{q}_{j}\right)=\frac{\partial \mathbf{C}}{\partial \mathbf{q}}=\left[\begin{array}{cccc}
\mathbf{I} & -\mathbf{A}_{i} \overline{\overline{\mathbf{r}_{i}}} \overline{\mathbf{G}_{i}} & -\mathbf{I} & \mathbf{A}_{j} \overline{\overline{\mathbf{r}_{j j}}} \overline{\mathbf{G}_{j}} \\
0 & -\mathbf{A}_{i} \overline{\overline{\boldsymbol{\omega}_{i}}} \overline{\mathbf{G}_{i}} \cdot \mathbf{A}_{j} \overline{\boldsymbol{\omega}_{j 1}} & 0 & -\mathbf{A}_{i} \overline{\overline{\boldsymbol{\omega}_{i}}} \cdot \mathbf{A}_{j} \overline{\overline{\boldsymbol{\omega}_{j 1}}} \overline{\mathbf{G}_{j}} \\
0 & -\mathbf{A}_{i} \overline{\overline{\boldsymbol{\omega}_{i}}} \overline{\mathbf{G}_{i}} \cdot \mathbf{A}_{j} \overline{\boldsymbol{\omega}_{j 2}} & 0 & -\mathbf{A}_{i} \overline{\boldsymbol{\omega}_{i}} \cdot \mathbf{A}_{j} \overline{\overline{\boldsymbol{\omega}_{j 2}}} \overline{\mathbf{G}_{j}}
\end{array}\right]=\left[\begin{array}{ll}
\mathbf{C}_{\mathbf{q}_{i}} & \mathbf{C}_{\mathbf{q}_{j}}
\end{array}\right]
$$

\section{Dynamics Model of MAAV-VT System}

The dynamics model of the MAAV-VT system is the reflection of its real service status. The instructions of control objectives adopted from the feasible path planning layer are transferred to the dynamics model. The control performances of hub motors and the mechanical characteristics of articulated mechanism between each vehicle are considered in the dynamics model. Thus, an integrated simulation platform is built to verify the accuracy of the path-following method raised above. The structure and parameters of each vehicle element are nearly the same as the electromobiles with the characteristics of four-wheel active steering and all-wheel-drive. Each element is constituted of four independent hub motors and two sets of steering mechanisms. The vehicle elements are articulated by a series of hinges and formed a unified whole. Thus, the dynamics model of each vehicle is studied first. Then, taking into account of the connection mechanism between each vehicle, the modeling method based on loop variables is used.

As is shown in Figure 4, the articulated mechanism is constituted of kinematic pairs and force elements. The articulated mechanism should satisfy the needs of connection force and the freedom of motion between each vehicle element. Taking the spring-damper system as an example, the mechanical properties of the articulated mechanism are analyzed.

The function of spring-damper system here is used to decrease the impact of longitudinal impulse between each vehicle. The acting points of the force element system in the front and rear vehicle element are recorded as $S_{i}$ and $S_{j}$. As is shown in Equation (4), the distance vector $\mathbf{r}_{S_{i} S_{j}}$ between $S_{i}$ and $S_{j}$ could be calculated by the position vector:

$$
\begin{aligned}
& \mathbf{r}_{S_{i} S_{j}}=\mathbf{R}_{i}+\mathbf{A}_{i} \overline{\mathbf{r}_{i S_{i}}}-\left(\mathbf{R}_{j}+\mathbf{A}_{j} \overline{\mathbf{r}_{j S_{j}}}\right), \\
& \dot{r}_{S_{i} S_{j}}=\mathbf{R}_{i}-\mathbf{A}_{i} \tilde{\mathbf{r}_{S_{i}}} \overline{\mathbf{G}} \dot{\theta}_{i}-\left(\mathbf{R}_{j}-\mathbf{A}_{j} \tilde{\mathbf{r}_{S_{j}}} \overline{\mathbf{G}} \dot{\theta}_{j}\right),
\end{aligned}
$$

where $\dot{r}_{S_{i} S_{j}}$ is the velocity vector and $\dot{\theta}_{j}$ is the first-order derivative of the attitude in the generalized coordinate. Thus, the acting force $\mathbf{F}_{S}$ could be expressed as follows:

$$
\mathbf{F}_{S}=\mathbf{K}\left(\mathbf{r}_{S_{i} S_{j}}-\mathbf{r}_{S_{i} S_{j}}^{0}\right)+\mathbf{C}\left(\dot{r}_{S_{i} S_{j}}\right)
$$




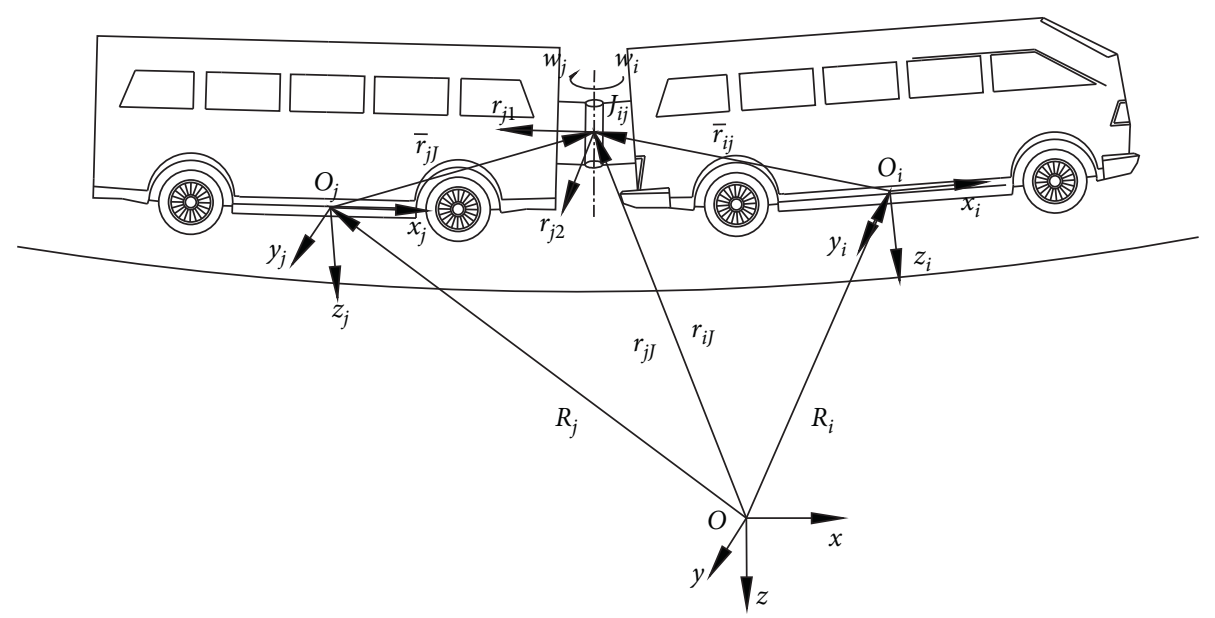

FIGURE 3: Kinematics constraints of the articulated mechanism.

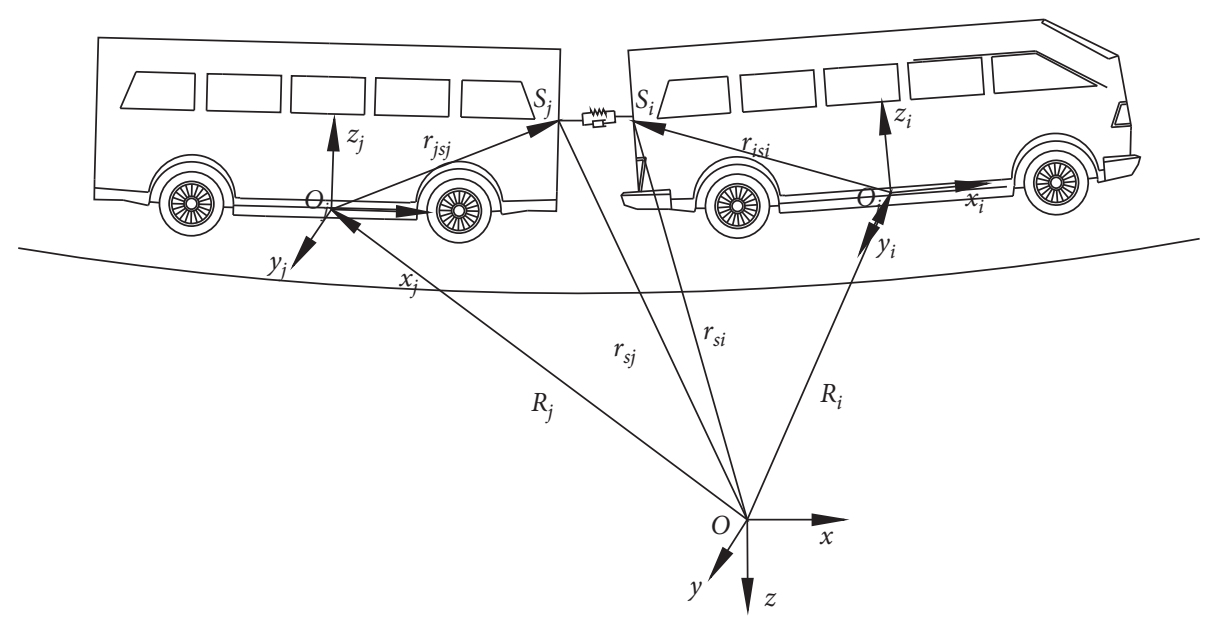

FIgURE 4: Spring-damper system.

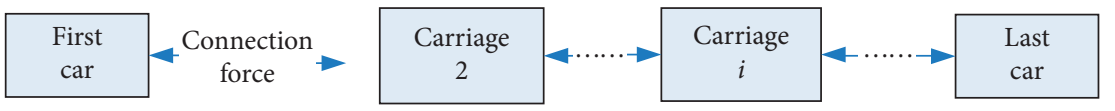

Figure 5: The calculation method of long-large train based on the loop variable.

where $\mathbf{K}$ and $\mathbf{C}$ are the stiffness matrix and the damping matrix separately. $\mathbf{r}_{S_{i} S_{j}}^{0}$ is the initial distance vector. The form of generalized force vector could be described as follows:

$$
\mathbf{Q}_{i}=-\mathbf{Q}_{j}=\left[\begin{array}{c}
\mathbf{F}_{S} e_{q} \\
\overline{\mathbf{G}^{T}} \mathbf{A}_{i}^{T} \widetilde{\mathbf{r}^{T}}{ }_{S_{i}} \mathbf{F}_{S} e_{q}
\end{array}\right] .
$$

The multi-articulated vehicle should consider the influence of the connection between each conjoint element. As is shown in Figure 5, each vehicle element is regarded as a basic unit which is the same with the dynamic model above in the modeling. Then, the connection forces are taken into account. The modeling method of long-large train based on the loop variable developed by CHI [30] is used here. The basic idea of this method is to regard each vehicle element as central integral unit. The kinematic equation could be expressed as

$$
\mathbf{M} \ddot{X}+\mathbf{C} \dot{X}+\mathbf{K X}=\mathbf{P},
$$

where $\mathbf{M}, \mathbf{C}$, and $\mathbf{K}$ represent the mass matrix, damping matrix, and stiffness matrix of the vehicle element. $\ddot{X}, \dot{X}$, and $\mathbf{X}$ are the generalized acceleration vector, velocity vector, and displacement vector. $\mathbf{P}$ is the generalized load vector.

Equation (8) changes to (9) while considering the acting forces $\mathbf{F}$ between each vehicle element:

$$
\mathbf{M} \ddot{X}+\mathbf{C} \dot{X}+\mathbf{K X}=\mathbf{P}+\mathbf{F} .
$$

Equation (9) is expanded for each basic integral unit, which is expressed as follows:

$$
\mathbf{M}_{\mathbf{i}} \ddot{X}_{\mathbf{i}}+\mathbf{C}_{\mathbf{i}} \dot{X}_{\mathbf{i}}+\mathbf{K}_{\mathbf{i}} \mathbf{X}_{\mathbf{i}}=\mathbf{P}_{\mathbf{i}}+\mathbf{F}_{\mathbf{i}}
$$

In this way, the integral of the whole multi-articulated vehicle is divided into small integral units. While giving the initial 
value and acting force of each vehicle element, the dynamic models of the whole multi-articulated vehicle could be built.

\section{Feasible Path Planning}

The aims of feasible path planning are to generate a feasible path of the multi-articulated vehicle which meeting the requirement of kinematics constraints, boundary conditions, and characteristic of actuators. The problem of path planning could be represented as the planning of a series of movement attitudes and gestures between the original state and the terminal state. The multi-articulated vehicle could operate according to the planning gestures to achieve the goal of path following.

5.1. Comparisons of the Path Planning Methods. Three kinds of path planning methods are compared, including spline curve fitting, Bessel curve fitting, and polynomial fitting. As shown in Figure 6, the results of cubic spline interpolation, quartic B-spline interpolation, and polynomial interpolation are relatively similar, with a high degree of coincidence. The main difference of each interpolation curve is reflected in the beginning end. It can be seen from the local enlarged view of the curve that the cubic spline interpolation curve has the smoothest transition, followed by B-spline. In order to make the polynomial interpolate each interpolation point, the degree of polynomial is higher to seven degree. The transition of the curve in this section is less gentle than that of cubic spline and B-spline. In addition to the initial node and the target node, the fitting curve obtained by using the Bezier function does not pass through other control points, which are only used to control the shape of the fitting curve. Therefore, the curve fitted by the Bezier function is the smoothest, but the disadvantage is that the function value of each control point and the tangent direction cannot be strictly controlled.

The curvature radius, slope, curvature value, and change rate of curvature value obtained by each method are shown as follows. As shown in Figure 6(a), the slope change of each curve is relatively gentle, and the results of cubic spline curve interpolation, B-spline interpolation, and seventh polynomial interpolation are relatively similar, and the slope change of Bessel fitting curve is the least. The curvature radius of each curve has little difference, and each curve generation method can better meet the requirement of the minimum curvature radius. The curvature radius of each curve generated from the above initial position to the target position is all greater than $10 \mathrm{~m}$. As can be seen from Figure 6(c), the curvature of each curve changes continuously, so the curves generated by each method can all realize the constraint conditions on the rate of curvature change proposed by the aforementioned actuator characteristics. As shown in Figure 6(d)), the curve curvature change rate of Cubic Spline interpolation and B-Spline interpolation did not show large peaks and troughs but showed a stable change trend, which was more conducive to meeting the requirement of curvature radius change rate. The interpolation method of seventh degree polynomial had a larger curvature change rate at the boundary.
Thus, considering from the feasible path constraint, cubic spline curve interpolation and B-spline interpolation can basically meet the requirements of planning and feasible path. The planned path meets the requirements of tracking point function values with continuous second derivative and controls the minimum curvature radius and the maximum radius of curvature change rate better. However, the first derivative value, namely, the trace point velocity direction, cannot be controlled. The higher order polynomial can satisfy the requirement of function value and derivative value at the control point, but the curvature change rate of the generated curve is difficult to guarantee. Therefore, combining the advantages of piecewise function and polynomial interpolation, a piecewise quartic polynomial interpolation method is selected to generate feasible paths for self-guided tram.

\subsection{Piecewise Quartic Polynomial Interpolation Method.} The physical quantities which describe the motion of multiarticulated vehicle are labelled as set $\mathbf{C}$. The set contains the location coordinates of each trace point $\left(x_{O_{b m}}, y_{O_{b m}}\right)$, the instantaneous turn center $\left(x_{O_{i}}, y_{O_{i}}\right)$, and the articulated mech$\operatorname{anism}\left(x_{J_{i}}, y_{J_{i}}\right)$. The attitudes, yaw velocity of each vehicle element, steering angle, and velocity of each wheel which recorded separately as $\varphi_{i}, \omega_{i}, \delta_{i j k}$, and $v_{i j k}$ are all included. The mapping function which is labelled as $\Gamma$ from the planned path to these physical quantities which control the motion features of the multi-articulated vehicle could be written as follows:

$$
\left\{\left(x_{O_{i}}, y_{O_{i}}\right), \varphi_{i}, \omega_{i}, \delta_{i j k}, \vec{v}_{J_{i}},\left(x_{J_{i}}, y_{J_{i}}\right)\right\}=\Gamma\left\{g(x), \vec{v}_{O_{b m}},\left(x_{O_{b m}}, y_{O_{b m}}\right)\right\} \text {, }
$$

where $g(x)$ is the planned path and $\vec{v}_{O_{b m}}$ is the velocity of each trace point. Parameter $m$ is the number of trace points. Parameter $i$ is the number of vehicle elements. Parameter $j$ and $k$ represent the location of each wheel, which are assigned as $f$ or $r$ on behalf of front and rear and $l$ or $r$ on behalf of left and right Figure 7.

Cubic spline and B-Spline interpolation are appropriately considered the kinematic characteristics of the multiarticulated vehicle. The two curves could fit the function values in trace points. And, they are all second differentiated to control the radius of curvature. These advantages are good to control the continuity of the path. However, the firstorder derivative is uncontrollable which means the direction of the curve is uncertain. The higher-degree polynomial is all right to control both the value and the first-order derivative in trace points, but the curvature of planned curve is hard to guarantee. Thus, a method of segmental interpolation based on quartic polynomial is proposed here. follows:

The polynomial equation in each segment is expressed as

$$
P_{h}(x)=\sum_{j=0}^{4} a_{h j} x^{j}
$$

Thus, the first- and second-order derivatives are expressed as the following equations: 


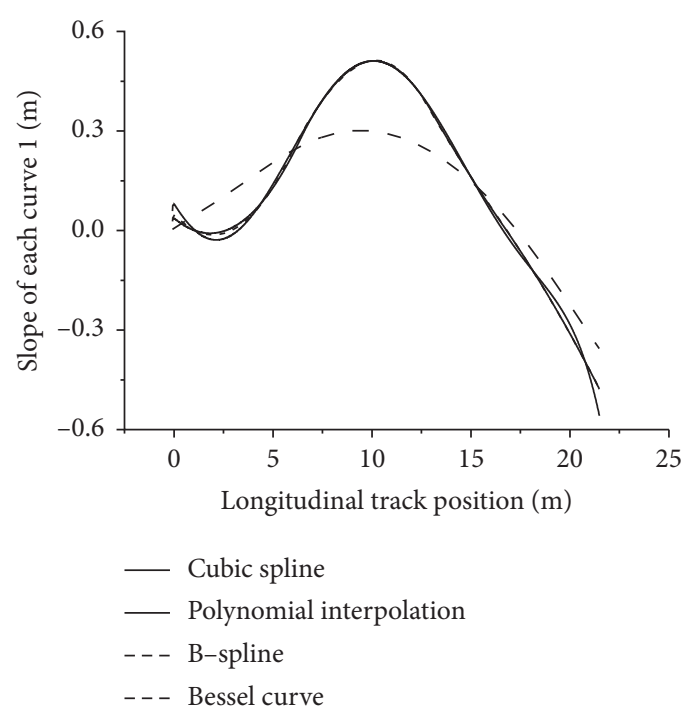

(a)

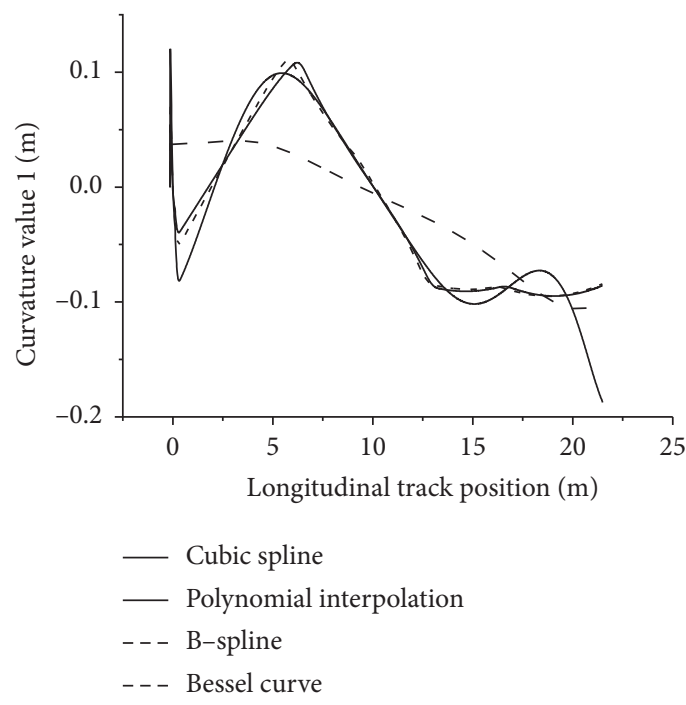

(c)

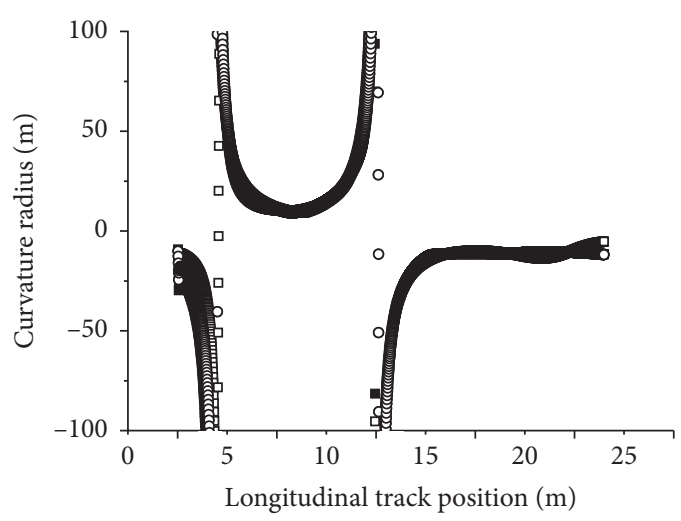

- Cubic spline

- Polynomial interpolation

- B-spline

- Bessel curve

(b)

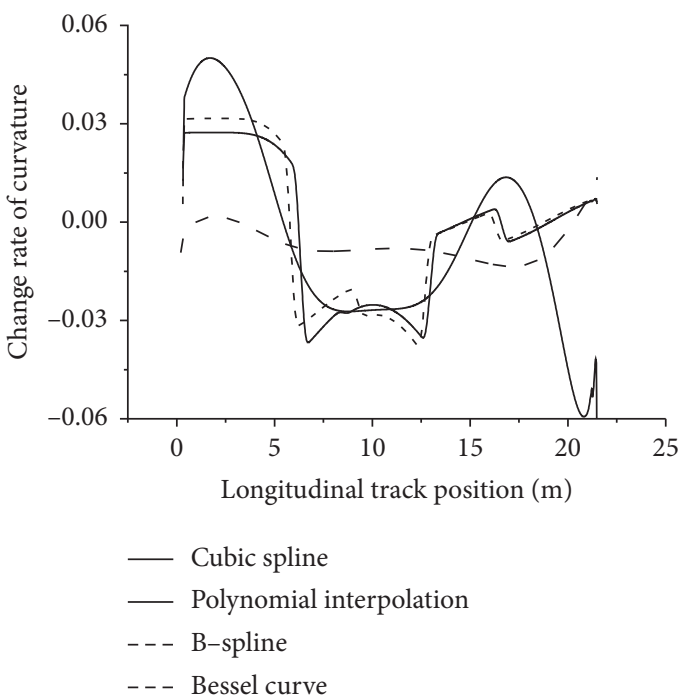

(d)

FIgURE 6: Comparison of results of different curve generation algorithms: (a) slope of each curve, (b) curvature radius, (c) curvature value, (d) change rate of curvature.

$$
\begin{aligned}
& P_{h}^{\prime}(x)=\sum_{j=1}^{4} j \cdot a_{h j} x^{j-1}, \\
& P_{h}^{\prime \prime}(x)=\sum_{j=2}^{4} j \cdot(j-1) \cdot a_{h j} x^{j-2} .
\end{aligned}
$$

The characteristics of the segmental interpolation based on quartic polynomial should guarantee the continuity of function values and the first and second derivatives in the boundary points.

\section{Verification of the Trajectory Tracking Strategy}

The coordinate trajectory tracking strategy of the multiarticulated vehicle based on the circulation of feasible path planning is verified by the constructed simulation platform. The simulation platform is built based on the dynamics model and trajectory calculation model. The values of control variables are calculated according to the replanned path and then transferred to the dynamics model. The actual motion trajectory and the kinetic parameters are calculated. The operation velocity is controlled by the first trace point which is preset at a medium constant value.

The trajectory of lemniscate is preset as the virtual track. This kind of track is always used in vehicle handling and stability testing. It is suitable to verify the trajectory tracking ability of the multi-articulated vehicle considering the executability and stability. The equation of the preset track is shown in the following equation:

$$
l=60 * \sqrt{\cos (2 \psi)} .
$$




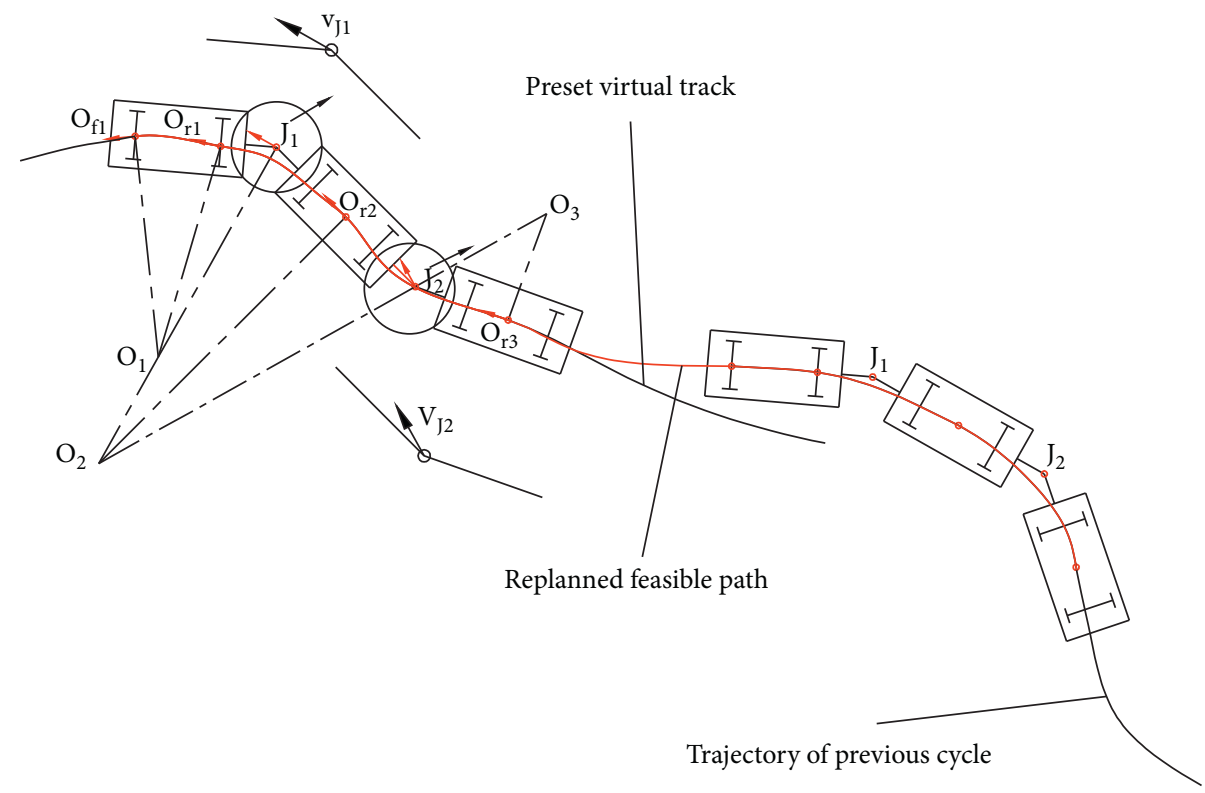

Figure 7: Feasible path planning.

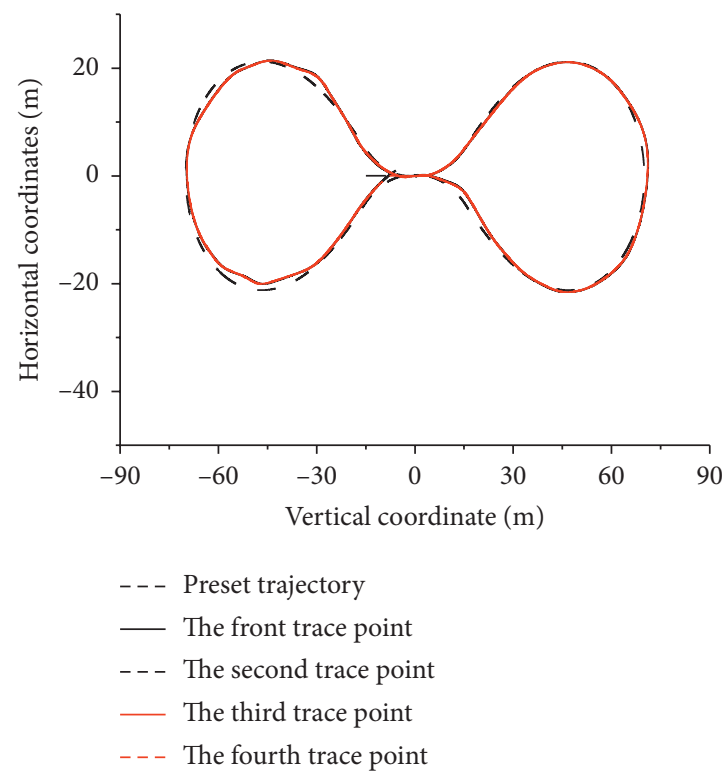

(a)

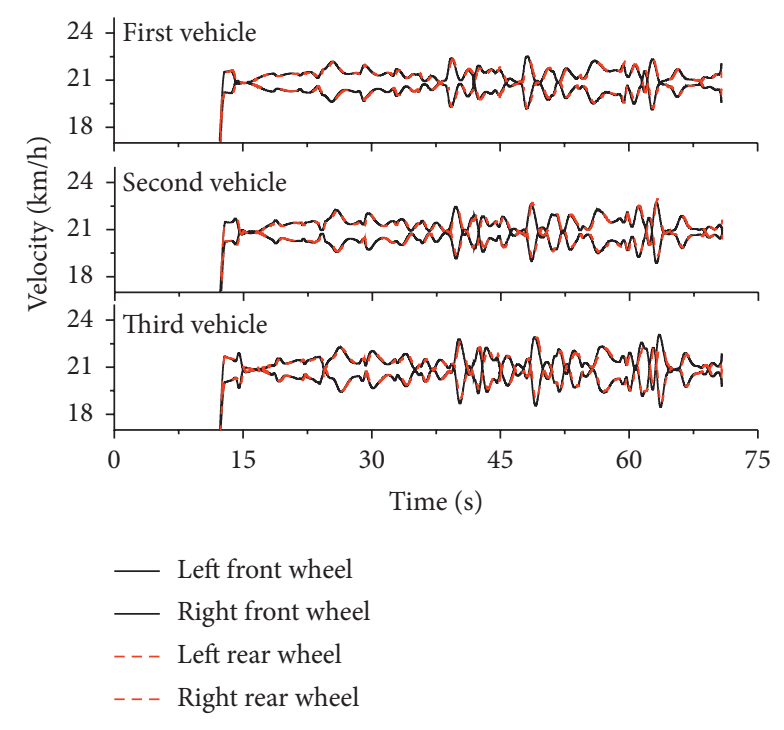

(b)

FIGURE 8: The comparison of preset and motion trajectory and the velocity of each wheel.

The minimum radius of the lemniscate is 20 meters while $\psi=0^{\circ}$. There is a transition curve before the lemniscate track. The transverse span and longitudinal span of the track are about 140 meters and 40 meters separately.

The black thick dash line represents the preset lemniscate track, and the others are the actual trajectory each trace point moved. As is shown in Figure 8, the trajectory of each vehicle is highly consistent with the preset track. The controller has detected sixteen times of the condition that the position or attitude error exceeded the preset threshold value. Under the circumstances, the controller would conduct the command of feasible path replanning to adjust the position and attitude to follow the guidance in front of the virtual track. The speed of the first trace point is constant at $6 \mathrm{~m} / \mathrm{s}$. The actual speed of each wheel controlled by hub motors is exported as Figure 8 . The fluctuation of the velocity curves agrees with the time of path replanning which means that the hub motors execute as control instructions. The multi-articulated vehicle moved at the right side of the lemniscate first. The processes of path replanning for trajectory tracking are increased obviously. As a result, the velocity curves and the actual trajectory of the multi-articulated vehicle are unsmooth than that of the left side. The whole variant trend of the velocity curves of each vehicle is nearly the same. However, there is a difference of 


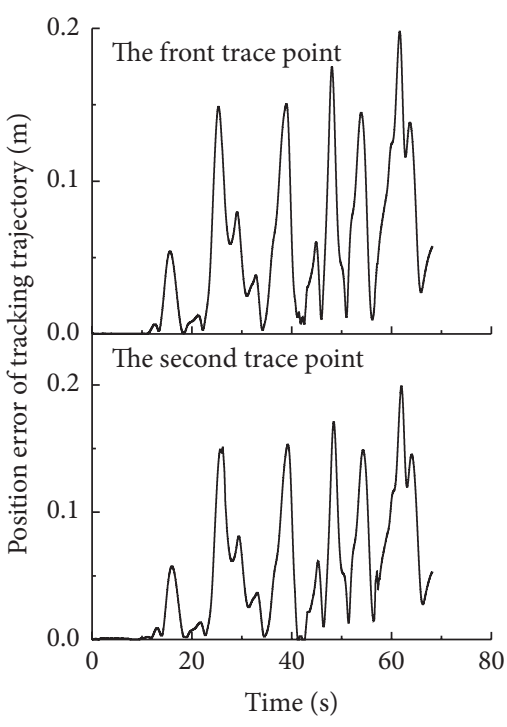

(a)

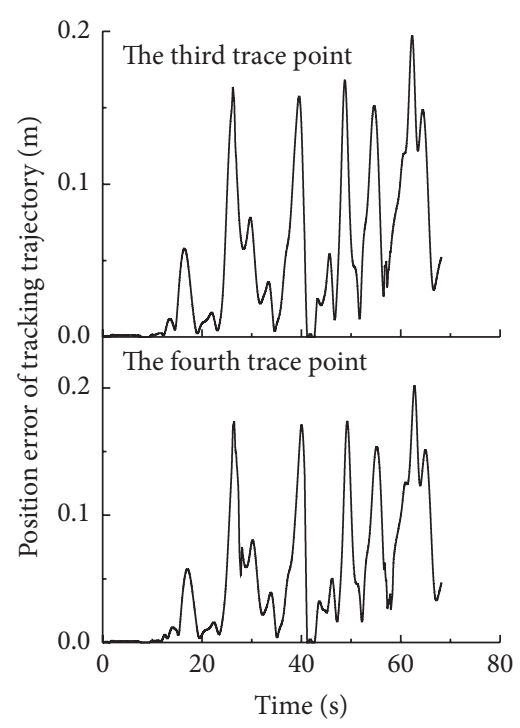

(b)

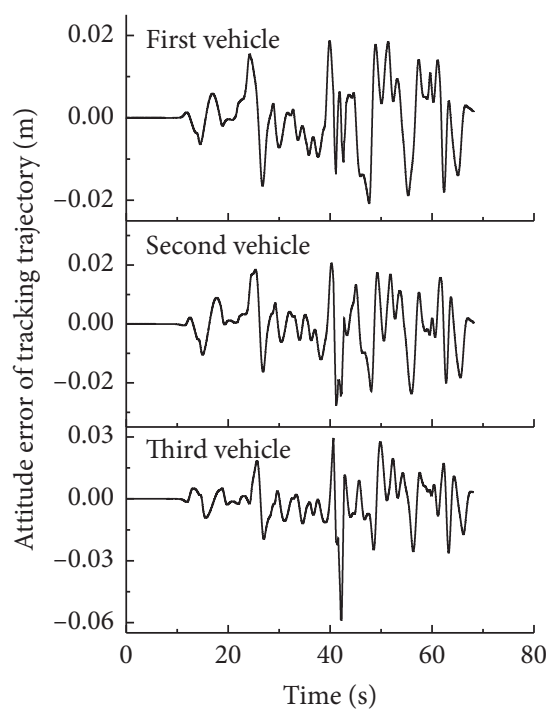

(c)

Figure 9: The position and attitude error of tracking trajectory.

time phase to guarantee each vehicle element to path through the planned feasible curve in turns.

The trajectory tracking error including the position error of each trace points and the attitude error of each vehicle is displayed in Figure 9. The variant trend of each trace points in different vehicle elements is nearly the same which proved great following features of the whole multi-articulated vehicle. The position error and the attitude error are fluctuated within 0.2 meter and $0.02 \mathrm{rad}$. The tracking error could also show that the tracking performances of the first half of the preset lemniscate track are better than that of the second half.

\section{Conclusions}

A new kind of modern public transportation vehicle named Multi-Articulated Guided Vehicle based on Virtual Track (MAAV-VT) is described in this article. It is a fusion of the operation model of urban rail transit and advance automotive technology. The following works are conducted in this article centered on the vehicle system:

1. The design concepts and general technologies of the MAAV-VT are generalized, which concludes using rubber tire support to simplify the construction, virtual track guide to realize self-guide, permanent magnet in-wheel motor drive to make each wheel independent, and mixed road rights to increase efficiency.

(2) As the core technology of the multi-articulated guided vehicle, the feasible path planning method based on the kinematics model of MAAV-VT is analyzed. The expected position determination method of MAAV-VT is proposed first to locate the vehicle. Then, the boundary constraint conditions are analyzed, and the curve generation method is proposed to generate feasible path of the whole vehicle. Finally, the trajectory tracking based on the circulation of feasible path planning is proposed. The circulation condition and terminal boundary of the circulation are analyzed.

(3) The dynamics model of the MAAV-VT system is built to reflect its real service status and verify the trajectory tracking strategy. The results show that the coordinate traction control strategy of the multiarticulated vehicle based on the circulation of feasible path planning has fairly good effects in the preset lemniscate track.

\section{Data Availability}

No data were used to support this study.

\section{Conflicts of Interest}

The authors declare that they have no conflicts of interest.

\section{Acknowledgments}

This work began from the original idea of tutor Prof. Zhang Weihua who also provided a lot of technical support of this work. This work was supported by the Fundamental Research Funds for the Central Universities (3122018C035).

\section{References}

[1] M. Burke, "Problems and prospects for public transport planning in Australian cities," Built Environment, vol. 42, no. 1, pp. 37-54, 2016.

[2] A. Kersys, "Sustainable urban transport system development reducing traffic congestions costs," Engineering Economics, vol. 22, no. 1, pp. 5-13, 2015.

[3] B. Furman, S. Ellis, L. Fabian et al., "Automated transit networks (ATN): a review of the state of the industry and prospects for the future," MTI Report, pp. 12-31, 2015. 
[4] O. Michler, R. Weber, and G. Forster, "Model-based and empirical performance analyses for passenger positioning algorithms in a specific bus cabin environment," in Proceedings of the 2015 International Models and Technologies for Intelligent Transportation Systems (MT-ITS), pp. 200-208, IEEE, Budapest, Hungary, January 2015.

[5] E. D. Dickmanns, "Detailed visual recognition of road scenes for guiding autonomous vehicles," in Advances in Real-Time Systems, pp. 225-244, Springer, Berlin, Germany, 2012.

[6] T. Deng and J. D. Nelson, "Recent developments in bus rapid transit: a review of the literature," Transport Reviews, vol. 31, no. 1, pp. 69-96, 2011.

[7] J. F. Reid, Q. Zhang, N. Noguchi, and M. Dickson, "Agricultural automatic guidance research in North America," Computers and Electronics in Agriculture, vol. 25, no. 1-2, pp. 155-167, 2000.

[8] J. D. Will, F. A. C. Pinto, N. Noguchi et al., "Sensor fusion framework for heading determination using gps and inertial measurement," in Proceedings of the 2000 ASAE Annual International Meeting, Milwaukee, WI, USA, July 2000.

[9] Y. Xia, Z. Zhu, and M. Fu, "Back-stepping sliding mode control for missile systems based on an extended state observer," IET Control Theory \& Applications, vol. 5, no. 1, pp. 93-102, 2011.

[10] J. N. Bakambu, V. Polotski, and P. Cohen, "Heading-aided odometry and range-data integration for positioning of autonomous mining vehicles," in Proceedings of the 2000 IEEE International Conference, pp. 279-284, Anchorage, AK, USA, September 2000.

[11] C. Y. Chan, B. Bougler, D. Nelson, P. Kretz, H. S. Tan, and W. B. Zhang, "Characterization of magnetic tape and magnetic marker as a position sensing system for vehicle guidance and control," in Proceedings of the American Control Conference, Chicago, IL, USA, June 2000.

[12] D. M. Hopstock and L. D. Wald, "Wald, verification of field model for magnetic pavement marking tape," IEEE Transactions on Magnetics, vol. 32, no. 5, pp. 5088-5090, 1996.

[13] H. T. Søgaard, "Evaluation of the accuracy of a laser optic position determination system," Journal of Agricultural Engineering Research, vol. 74, no. 3, pp. 275-280, 1999.

[14] S. Se, D. G. Lowe, and J. J. Little, "Vision-based global localization and mapping for mobile robots," IEEE Transactions on Robotics, vol. 21, no. 3, pp. 364-375, 2005.

[15] G. Adorni, S. Cagnoni, S. Enderle et al., "Vision-based localization for mobile robots," Robotics and Autonomous Systems, vol. 36, no. 2-3, pp. 103-119, 2001.

[16] J. K. Rosenblatt, "DAMN: a distributed architecture for mobile navigation-thesis summary," Journal of Experimental and Theoretical Aitificial Intelligence, AAAI Press, vol. 9, no. 2-3, pp. 339-360, 1997.

[17] R. A. Brooks, "A robust layered control system for a mobile robot," IEEE Journal on Robotics and Automation IEEE Journal of Robotics and Automation, vol. 2, no. 1, pp. 14-23, 1986.

[18] M. Piaggio, "Non-hierarchical Hybrid Architecture for Intelligent robots," in Proceedings of ATAL: Workshop on Agent Theories Architectures and Languages, Paris, France, July 1998.

[19] G. N. Saridis, "Toward the realization of intelligent controls," Proceedings of the IEEE, vol. 67, no. 4, pp. 1115-1133, 2003.

[20] J. S. Albus, H. G. McCain, and R. Lumia, NASA/NBS Standard Reference Model for Telerobot Control System Architecture (NASREM), National Institute of Standards and Technology, Gaithersburg, MD, USA, 1989.
[21] T. Le-Anh and M. B. M. De Koster, "A review of design and control of automated guided vehicle systems," European Journal of Operational Research, vol. 171, no. 1, pp. 1-23, 2006.

[22] J. H. Xin, S. M. Li, Q. B. Liao et al., "The application of fuzzy logic in exploration vehicle," in Proceedings of the 4th International Conference on Fuzzy Systems and Knowledge Discovery, vol. 4, pp. 199-203, Haikou, China, August 2007.

[23] J. Wang, J. Steiber, and B. Surampudi, "Autonomous ground vehicle control system for high-speed and safe operation," in Proceedings of the 2008 American Control Conference, pp. 218-223, Seattle, WA, USA, June 2008.

[24] J. Wit, C. D. Crane, and D. Armstrong, "Autonomous ground vehicle path tracking," Journal of Robotic Systems, vol. 21, no. 8, pp. 439-449, 2004.

[25] M. H. Hebert, C. Thorpe, and A. Stentz, Intelligent Unmanned Ground Vehicles:autonomous Navigation Research at Carnegie Mellon, Springer Science \& Business Media, Berlin, Germany, 2012.

[26] R. Olfati-Saber, "Global configuration stabilization for the VTOL aircraft with strong input coupling," IEEE Transactions on Automatic Control, vol. 47, no. 11, pp. 1949-1952, 2002.

[27] J. Chen, Z. Shuai, H. Zhang, and W. Zhao, "Path following control of autonomous four-wheel-independent-drive electric vehicles via second-order sliding mode and nonlinear disturbance observer techniques," IEEE Transactions on Industrial Electronics, vol. 68, no. 3, pp. 2460-2469, 2021.

[28] A.-T. Nguyen, C. Sentouh, H. Zhang, and J.-C. Popieul, "Fuzzy static output feedback control for path following of autonomous vehicles with transient performance improvements," IEEE Transactions on Intelligent Transportation Systems, vol. 21, no. 7, pp. 3069-3079, 2020.

[29] Q. Shi and H. Zhang, "Fault diagnosis of an autonomous vehicle with an improved SVM algorithm subject to unbalanced datasets," IEEE Transactions on Industrial Electronics, p. $1,2020$.

[30] C. H. I. Mao-Ru, J. Yi-Ping, Z. Wei-Hua et al., "System dynamics of long and heavy haul train," Journal of Traffic and Transportation Engineering, vol. 11, no. 3, pp. 35-40, 2011. 